\title{
Interactive comment on "Rainfall intensity bursts and the erosion of soils: an analysis highlighting the need for high temporal resolution rainfall data for research under current and future climates" by David L. Dunkerley
}

Anonymous Referee \#2

Received and published: 25 February 2019

\section{Summary}

This paper presents an argument for measuring rainfall at higher temporal resolution (preferably sub-hourly) than is typically available in contemporary precipitation datasets (where daily or at best hourly rainfall records available) in order to capture "intensity bursts," or short periods of potentially extremely intense rainfall, that may exert a much stronger control on soil removal/transport events than mean rainfall intensity derived from longer averaging periods. A broad overview of erosion and transport mechanisms directly affected by precipitation is given in the introduction and discussion sections,

Printer-friendly version

Discussion paper 
while the main results of the paper pertain to describing intermittency and the increasing relationship between rainfall intensity and temporal resolution using time series of precipitation measurements from two field sites in Australia. I find the description of intermittency using the two rainfall datasets to be rather qualitative in nature. The author cites several studies (e.g. Paschalis et al., 2014; Monjo, 2016; Beranova et al., 2018; Pohle et al., 2018; Schleiss, 2018) that provide a more quantitative view of intermittency (using, e.g., multifractal analysis), but chooses not to apply the tools used in those studies in this manuscript. These analytical techniques are sufficiently complex that I understand the author may not have wished to complicate the study with a lengthy discussion of the relevant mathematical framework, although I do think the author's argument would be more convincing with a more rigorous quantification of intermittency or the impact of intermittency on predicted erosion and transport rates.

While I agree with the author's primary thesis that using precipitation measurements at hourly or daily aggregation leads to significant underestimation of process rates relevant to erosion or sediment transport, I don't see a specific suggestion for how precipitation should be measured to more accurately represent the physics at play. My main concern is that temporal intermittency is addressed without paying comparable attention to spatial intermittency. In essence, I don't see why having greater temporal resolution from point measurements (be it from rain gauge, disdrometer, etc.) necessarily leads to a better understanding of plot or catchment scale erosion/sediment transport. Even over an extremely homogeneous land surface, intermittency is generated in clouds aloft due to their close coupling with atmospheric turbulence, and surface rainfall likewise exhibits significant intermittency. Accounting for this spatial intermittency seems just as important the temporal aspect; indeed, this very issue has been explored extensively by the hydrology community (e.g. Berne et al., 2004). I recognize that such a broad study was not the point of the present article; I bring all of this up primarily so the author is aware that there exists a large body of work relating to these questions.

\section{ESurfD}

Interactive comment
Printer-friendly version

Discussion paper 
Overall, the paper attains its stated purpose: to illustrate the intermittency of precipitation (i.e. the magnitude of rainfall intensity maxima increase as measurements are disaggregated) and highlight the potential impacts on earth surface processes. I have some concerns about the presentation of results, but I can without hesitation recommend this article for publication with minor revisions.

Please find below other general comments, followed by specific comments. Specific comments refer to "Pxx, Lyy" where $P$ is followed by a page number and $L$ by line number.

General comments:

* A major question for me regarding the measurements presented is whether buckets with different tipping volumes can be directly compared using "inter-tip time." The manuscript indicates that "raw" ITTs are analyzed - is any normalization performed to account for different bucket volume? It's not quite comparing apples and oranges (perhaps more akin to apples and pears), but I think it is important to quantify what the impact on ITT run statistics would be if, say, MM had a $0.5 \mathrm{~mm}$ capacity instead of 0.2 $\mathrm{mm}$ to understand whether bucket volume affects diagnosed intermittency.

* Modified Julian dates are convenient for running the analysis, but not for conveying the results. Consider plotting a more intuitive time format (i.e., HH:MM:SS with yyyy/mm/dd date below) so the reader can easily see that sub-hourly variability is important. This is not to say that your use of MJD is misguided, simply that the points you are trying to convey will be "punchier" if readers don't have to spend mental effort decoding what a fraction of an MJD means.

${ }^{*}$ Regarding the use of tables in Section 2.2: Tables 2 and 3 are very difficult to understand in their present form. There are 7 columns of data and comparing the two very large tables to understand differences between the MM and FG sites is nontrivial. I would prefer to see these data presented graphically, perhaps even in one unified log$\log$ axis figure. This would also enable you to reduce the amount of data presented,

ESurfD

Interactive comment
Printer-friendly version

Discussion paper 
e.g. "number of tips in longest run" and "rain depth of longest run" are related by a simple multiplicative constant, and number of tips is subject to the bucket volume issue discussed above such that I don't think the two sites can be easily and directly compared using that metric. Conversely, the statistics given in the first two paragraphs of this section (average rain day accumulation and intensity, hourly intensity, various percentile values of intensity, etc.) would be well suited to tabular presentation, especially considering that one of the author's main points is to contrast conditions at the two sites considered. Regarding the percentile values, the cumulative distribution functions of rainfall intensity at hourly/daily aggregation could also be presented in graphical format for ease of comparison.

* The threshold given for "extreme" precipitation in Tokay and Short (1996) is $20 \mathrm{~mm}$ $\mathrm{h}-1$ as opposed to the $30 \mathrm{~mm} \mathrm{h-1}$ value used in the manuscript. While I suspect the exact choice of what constitutes "extreme" intensity is likely somewhat unimportant, what fraction of $M M$ and $F G$ rain at $1 \mathrm{~s}$ resolution falls in this category using the Tokay and Short definition of $\mathrm{R}>20 \mathrm{~mm}$ h-1? Is there a reason you used a modified criterion?

* What is the end goal of increasing temporal resolution? Is it to resolve individual raindrop collisions with the ground? Or to have some "ideal" sampling of the rain rate distribution (the definition of "ideal" being a subjective determination)? Or something else entirely? As stated above, I agree with the author that hourly/daily aggregation is of limited relevance for characterizing erosion, or really any other process that is highly nonlinear and therefore driven by extreme values, but there are other considerations that inform how temporal resolution is chosen for precipitation rate measurements than aggregation for the convenience of reducing data amount. In this context, it may be seen as a tradeoff: having increased knowledge of the "instantaneous" rain rate at a single "point" or rain gauge may be offset by less confidence that the instantaneous measurement at a given gauge is representative of the rain rate over some broader spatial area of interest (e.g. plot scale) precisely because of the intermittency aspect of rain.

\section{ESurfD}

Interactive comment
Printer-friendly version

Discussion paper 
* Section 4.1 is a great argument for having higher resolution rain rate measurements, but there's still quite a disconnect between the 5-10 minute fixed clock intensities cited here versus the $1 \mathrm{~s}$ measurements presented. Is this intentional?

Specific comments:

P3, L22: Peters and Christensen (2002) citation not in bibliography

Interactive

comment

P5, L19/23: Are the "mean annual rainfall" figures given simply the average over the observational records analyzed in the manuscript? If so, please explicitly state that the averages are over the observational period and not derived from some longer term record. It would be inaccurate to frame these as climatological means, especially given the author's comments regarding the large variability of annual rainfall at the FG site.

P6, L16: What is the sensitivity of the results to the choice of MIT? Is a single value of MIT valid for two different sites? What I'm getting at is that you've set this number as if it were a universal constant, while different storm systems may have different characteristic inter-event times. In any case, this is more something that I'm curious about than a comment that calls for rigorous sensitivity testing.

P9, L15-19: The data presented here would be more digestible if also given in a table.

P10, Figure 2: Can these three curves be presented with differing line style or color in a single figure? It would starkly highlight the loss of magnitude due to coarse-graining the measurements thereby giving greater weight to your argument. If this is undesirable, I am interested to hear your argument as to why.

P11-12, Tables 4-5: As with Tables 2-3, this data could be much more concisely expressed in graphical form.

P17, L25: Typo, "relatiobship" approximately halfway through line.

P19, L6: Insert commas in "Hubbert. . reported I10 I30 and I60 for. ..."

P19, L14: "In catchments in SW Germany" (add "in" after "catchments") 
P20, L18: Should "Thus" be "This?"

Interactive comment on Earth Surf. Dynam. Discuss., https://doi.org/10.5194/esurf-2018-94, 2019.

\section{ESurfD}

Interactive comment 